\title{
El entrenamiento físico mejora modestamente el estado de salud percibido por pacientes con insuficiencia cardiaca
}

\author{
Exercise training improves modestly health status perceived by patients with heart failure
}

\begin{abstract}
Objetivo
Evaluar los efectos del entrenamiento sobre el estado de salud percibido por los pacientes con insuficiencia cardiaca (IC) crónica sumado al tratamiento médico basado en la evidencia. El reportado en este trabajo era un objetivo secundario de un estudio que había sido diseñado para evaluar sus efectos sobre la mortalidad y el número de internaciones, y que no pudo hallar diferencias significativas entre ambos grupos ${ }^{1}$.
\end{abstract}

\section{Diseño, lugar y pacientes}

Ensayo clínico aleatorizado y controlado desarrollado en 82 centros de EE.UU. Canadá y Francia. Fueron reclutados 2331 pacientes con IC y fracción de eyección del ventrículo izquierdo menor a $35 \%$ en clase funcional NYHA II-IV, dispuestos a realizar un entrenamiento físico y que estuvieran recibiendo tratamiento farmacológico óptimo basado en la evidencia.

\section{Intervención}

Además de recibir los cuidados habituales, los pacientes del grupo intervención fueron incluidos en un programa de entrenamiento de ejercicio aeróbico supervisado (tres veces por semana) durante el cual llegaban al $60-70 \%$ de la frecuencia cardiaca de reserva; prescribiéndoseles luego un entrenamiento domiciliario de la misma intensidad para ser realizado cinco veces por semana. La aleatorización fue estratificada según el centro y la etiología de la IC.

\section{Medición de los resultados principales}

El estado de salud se evaluó mediante el cuestionario autoadministrado $\mathrm{KCCQ}^{2-3}$, de 23 ítems y dividido en subescalas de limitaciones físicas, calidad de vida y limitaciones sociales. Se valora en una escala de 0 a 100 puntos y fue administrado en la primera visita, cada tres meses durante los primeros $12, \mathrm{y}$ anualmente hasta los cuatro años de seguimiento. Los resultados fueron analizados por intención de tratar, considerándose significativa una diferencia de cinco puntos o más.

\section{Resultados principales}

En ambos grupo se observó a los tres meses una mejoría inicial del puntaje total y en las subescalas, algo más importante en el grupo entrenamiento, aun luego de los ajustes multivariables $^{*}$ y los análisis de subgrupos*. Esta mejoría se mantuvo estable a lo largo del seguimiento aunque sin diferencias significativas. Ver tabla 1.

Tabla 1: cambios estimados en el estado de salud reportado por pacientes con insuficiencia cardíaca (puntaje de 0 a 100).

\begin{tabular}{c|c|c|c|}
\hline & \multicolumn{2}{|c|}{ Diferencia de puntaje (IC95\%) } & \\
\hline $\begin{array}{c}\text { Escala KCCQ } \\
\text { Sumatoria total }\end{array}$ & $\begin{array}{c}\text { Cuidados } \\
\text { usuales }\end{array}$ & Entrenamiento & $\begin{array}{c}\text { Diferencia entre } \\
\text { grupos (IC95\%) }\end{array}$ \\
\hline A los tres meses & $3,28(2,28$ a 4,09) & $5,21(4,42$ a 6,00$)$ & $1,93(0,84$ a 3,01$)$ \\
A los cuatro años & $0,01(-0,05$ a 0,03$)$ & $0,00(-0,04$ a 0,03$)$ & $0,01(-0,05$ a 0,07$)$ \\
\hline
\end{tabular}

\section{Conclusiones}

Ambos grupos mejoraron modestamente inicialmente, algó más el de los pacientes sometidos a entrenamiento.

Palabras claves: insuficiencia cardíaca, entrenamiento físico, calidad de vida. Keywords: cardiac failure, exercise training, quality of life.

Fuentes de financiamiento: National Heart, Lung, and Blood Institute.

\section{Comentario}

Si bien suele implementarse el entrenamiento físico en los programas de manejo IC, estas terapéuticas no cuentan con óptima evidencia de sus efectos.

Por ejemplo, una revisión Cochrane ${ }^{4}$ concluyó que existe evidencia de que la rehabilitación mejora a corto plazo la calidad de vida de los pacientes con IC, requiriéndose estudios de mayor envergadura; mientras que otra revisión sistemática ${ }^{5}$ no halló benfición sobre el efecto de la prescripción de ejercicios en el domicilio. Si bien el estudio que hemos resumido reporta diferencias estadísticamente significativas, su implicancia clínica sería escasa. Cabe discutir si aporta luz sobre la indicación de rehabilitación aeróbica como parte del tratamiento de la IC, sin evidencia de que tenga efecto positivo sobre la mortalidad, el número de internaciones ni la calidad de vida. Además, como es de suponer, la imposibilidad de enmascarar la intervención podría sobrevalorar su efecto. Por otro lado, tampoco fueron documentados daños atribuibles a la intervención.

\section{Conclusión de la comentadora}

Aunque la mejoría hallada parece clínicamente irrelevante, la indicación de actividad física podría respaldarse sobre otros efectos benéficos atribuibles a la misma. El desafío pendiente es identificar alguna acción que involucre activamente a los pacientes para influenciar su adherencia al tratamiento integral y modificar el curso de su enfermedad.

Maia Burstein [ Servicio de Medicina Familiar y Comunitaria del Hospital Italiano de Buenos Aires. ] maia.burstein @ hospitalitaliano.org.ar

Burstein M. El entrenamiento físico mejora modestamente el estado de salud percibido por pacientes con insuficiencia cardiaca. Evid. Actual. Práct. Ambul; 12(3): 91, Jul-Sep 2009. Comentado de: Flynn K y col. Effects or Exercise Training on Health Status in Patiens with Chronic Heart Failure. HF-ACTION Randomized Controled Trial. JAMA 2009;301(14):1451-9.

PMID: 19351942. Disponible en URL: http://jama.ama-assn.org/cgi/content/full/301/14/1451

\footnotetext{
Referencia

1. O'Connor CM y col, for the HF-ACTION Investigators. Efficacy and safety of exercise training in patients with chronic heart failure : HF-ACTION randomized contorled trial: JAMA. 2009; 301(14);1439-1450.

2. Green C y col.Development and evaluation of the Kansas City Cardiomyopathy Questionnaire: a new health status measure for heart failure. J Am Coll Cardiol.

2000;35(5): 1245-55. 3. Spertus J y col. Carc

2005;150(4):707-715.
4. ReesK y col. Evidence based rehabilitation for Herat failure. Cochrane Database Syst Rev. 2004;(3):CD003331.

5. Chien C y col., Home-based exercise increases exercise capacity but not quality of life in people with chronic Heart failure: a systematic review. Aust J. Physiother: 2008;54(2):87-93.
} 